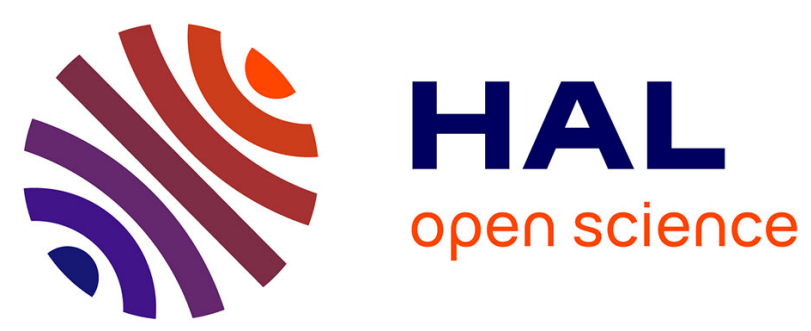

\title{
La fabrique du corps sexué
}

Laurie Laufer

\section{To cite this version:}

Laurie Laufer. La fabrique du corps sexué. Recherches en psychanalyse, 2010, Politique du sexuel, 10, pp.231-241. hal-01411214

\section{HAL Id: hal-01411214 https://hal.science/hal-01411214}

Submitted on 7 Dec 2016

HAL is a multi-disciplinary open access archive for the deposit and dissemination of scientific research documents, whether they are published or not. The documents may come from teaching and research institutions in France or abroad, or from public or private research centers.
L'archive ouverte pluridisciplinaire HAL, est destinée au dépôt et à la diffusion de documents scientifiques de niveau recherche, publiés ou non, émanant des établissements d'enseignement et de recherche français ou étrangers, des laboratoires publics ou privés. 


\section{Recherches en Psychanalyse}

10 | 2011 :

Politique du sexuel

\section{La fabrique du corps sexué}

\section{LAURIE LAUFER}

\section{Résumé}

En quoi la question de la sexualité est-elle importante dans la construction identitaire? En quoi l'image du corps peut-elle déterminer la construction d'une identité sexuelle? La fabrique d'un corps échappe-t-elle aux représentations de son temps? Depuis le XVIIème siècle, la sexualité et les pratiques sexuelles sont l'objet et l'enjeu d'un discours scientifique qui détermine l'identité d'un sujet. Dans ses travaux, Michel Foucault étudie le discours sur le sexe qui établit historiquement le lien entre la sexualité, la subjectivité et l'obligation de vérité, une triade qu'il verse au compte de la psychanalyse comme héritière de cette scientia sexualis qui cherche la vérité au fond du sexe. La psychanalyse a-t-elle perdu le tranchant subversif de son geste qui invente la sexualité infantile polymorphe?

\section{Entrées d'index}

Mots-clés : psychanalyse, Foucault, corps, genre, identité sexuelle, normes

\section{Texte intégral}

Comme le rappelait Foucault qui n’a pas toujours critiqué Freud, l'invention 1 de Freud s'est démarquée des théories de la dégénérescence, et à l'époque des Krafft-Ebing, des Kraepelin, des Morel, ce geste, notamment avec l'invention de la sexualité infantile, était véritablement subversif. Qu'en est-il aujourd'hui de ce geste subversif? La subversion de ce geste peut-elle se prolonger aujourd'hui dans nos pratiques?

Les deux «piliers » de la civilisation, que Freud considérait comme les deux grandes énigmes de la vie psychique, à savoir la mort et la sexualité ainsi que les pratiques corporelles auxquelles elle donne lieu, ne laissent pas de faire scandale. Depuis le XviI e siècle, la sexualité et ses pratiques, « cet obscur objet de désir», sont devenus objet d'un discours scientifique (médical, psychiatrique, juridique, politique) où le sujet est objectivé comme support de 
la connaissance. Il s'agit donc de «dire vrai » à propos de la sexualité et, comme l'écrit Foucault, « la sexualité, bien plus qu'un élément de l'individu qui serait rejeté hors de lui, est constitutive de ce lien qu'on oblige les gens à nouer avec leur identité sous la forme de la subjectivité1. » Dès lors, il s'agit de savoir ce qu'il en est de la vérité du sexe, la sexualité devenant un objet d'enquête spécifique pour le pouvoir et qui repose sur des discours de "véridiction », selon le terme de Michel Foucault.

«"Nous sommes notre sexualité", du moins nous l'a-t-on dit et redit » écrit Arnold Davidson. Il poursuit: «En ce sens, il n'y a aucun doute que cela va sans dire; nous ne saurions penser à nous-mêmes, à notre identité psychologique la plus fondamentale sans penser à notre sexualité, à cette couche souvent profonde et secrète de nos désirs qui révèle le genre d'individu que nous sommes. Et le "triomphe" des sciences humaines est précisément d'avoir mis en lumière, avec toute la force des concepts scientifiques, le rôle de la sexualité dans la formation de notre personnalité, sa place privilégiée au couur de notre vie psychique 2 . » Et Davidson, d'ajouter, citant Antoni Tapiès : «Il nous faut provoquer un mouvement qui "irrite" et, à la fois, peut faire réfléchir tous les bien-pensants qui se croient détenteurs de la vérité. [...] Et si la chance nous sourit, ce ne sera pas l'effet le moins significatif de ce travail que de produire cette friction qui nous permet d'établir un étrange et nouveau rapport à nous-mêmes ${ }^{3}$. »

2 En quoi la question de la sexualité est-elle importante dans la construction identitaire? Et l'est-elle toujours? En quoi l'image du corps peut-elle déterminer la construction d'une identité sexuelle? La fabrique d'un corps échappe-t-elle aux représentations de son temps?

\section{« Le doute visuel »}

Pour déplier ces aspects, je voudrais partir d'un épisode contemporain qui a fait grand bruit dans le monde sportif : Il s'agit de la mésaventure de Caster Semenya, athlète sud-africaine. L'affaire avait fait scandale lors des championnats du monde d'athlétisme en août 2009 à Berlin. Les experts s'interrogeaient en effet sur le « vrai sexe » de l'athlète sud-africaine qui avait remporté la finale féminine du $800 \mathrm{~m}$. Caster Semenya avait dû alors se plier à de multiples analyses sanguines et chromosomiques et avait subi des examens médicaux, des tests gynécologiques, des entretiens psychologiques. Selon le quotidien The Sydney Morning Herald Tribune, la championne du monde du $800 \mathrm{~m}$ serait hermaphrodite. Le journal australien explique en effet que les examens ordonnés par la Fédération internationale d'athlétisme ont « prouvé » que Semenya possédait à la fois des organes génitaux féminins et masculins. Les rapports médicaux indiquent que la jeune Sud-Africaine n'a pas d'ovaires, mais possède au contraire des testicules internes qui produisent de la testostérone et l'avantagent donc par rapport à ses concurrentes. À la rubrique des sports de différents quotidiens, on peut lire que « Pierre Weiss secrétaire général de la Fédération internationale d’athlétisme a tenu à préciser que des "tests de féminité" étaient actuellement réalisés par des médecins, en Allemagne et en Afrique du Sud, dont les résultats seraient connus dans deux ou trois semaines. "Oui, elle sera à la cérémonie des médailles, a dit Pierre Weiss. Il serait totalement injuste de l'exclure. Il n'y a pas de preuves qu'elle ne soit pas une femme, il y a juste un doute visuel. Pas suffisant pour radier l'athlète." ». 
L'athlète n'a jamais été interviewée elle-même, c'est le président de la fédération qui se fait son porte-voix. Le « doute visuel » ôte donc toute parole à la jeune femme. Voici ce que l'on a pu lire dans Le Monde daté du 20 Août 2009 :

« C'est l'inconnue du 800 mètres, l'invitée inattendue sur la plus haute marche du podium. Une invraisemblable [c'est moi qui souligne] gamine de 18 ans a plané, mercredi 19 août, sur la piste bleue des Mondiaux de Berlin. La SudAfricaine Caster Semenya a fauché l'or en 1'55"45, meilleure performance mondiale de l'année. La jeune surdouée a surclassé la tenante du titre, arrivée deux secondes plus tard.Une prodige? Avec sa mine adolescente, sa voix chaude, son duvet, ses muscles hors-normes (comparés au physique maigrichon de ses rivales) et sa démarche pleine de grâce... masculine, [sic], Caster Semenya jette le trouble sur sa féminité. Si la médaillée d'or était un homme? Ou un hermaphrodite? Cette question des plus sérieuses est l'objet de recherches de la Fédération sud-africaine et de la Fédération internationale d'athlétisme. »

4 Les sportives des pays de l'Est des années 1960, qui avaient pris de la testostérone, avaient incité la Fédération internationale d'athlétisme à réaliser des tests salivaires de féminité. En 1992, l'IAAF a décidé de stopper ces tests. « Ils n'étaient pas sûr à 100 \% », avait souligné M. Weiss. À cette époque, une Espagnole avait été radiée des épreuves d'athlétisme à la suite d'examens de l'IAAF révélant qu'il s'agissait d'un homme. Des tests ultérieurs avaient prouvé que l'athlète était bien de sexe féminin !

Du côté de la Fédération sud-africaine, et d'après ce que j'ai pu lire dans la presse qui a relaté cet événement, on considère que les choses sont allées trop loin, très vite. Leonard Chuene, le président de la fédération d’athlétisme s'emporte et défend sa jeune protégée :

« Malheureusement, les médias ont essayé de mettre une grosse pression. Mais nous continuons à la supporter parce que je crois qu'elle n'a pas commis d'acte criminel. Elle n'a pris aucune substance pour améliorer ses performances. Ce n'est pas une criminelle. Elle ne peut pas être criminelle à cause de son physique ou de sa façon d'être. Je n'ai jamais vu une personne être montrée du doigt sur la base d'un look ou d'un comportement naturel. Elle est désormais au sommet du monde. Elle est jeune et nous la soutenons. Nous lui faisons bien sentir qu'elle n'a rien fait de mal. Le pays est content d'elle. Elle nous a rendus fiers. Elle doit être très heureuse de sa performance ${ }^{4}$ ».

Aussi dans cet événement qui aurait pu être de l'ordre de l'anecdote, une jeune femme est-elle privée de sa propre parole. En vertu d'un « doute visuel » sur les caractères secondaires sexuels (musculature, duvet), on lui assigne un corps qui n'est pas le sien. Cette affaire rappelle le cas de l'Indienne Santhi Soundarajan. Cette athlète avait perdu sa médaille d'argent du 800 mètres, gagnée aux J eux asiatiques de Doha (Qatar), en 2006, après avoir échoué aux «tests de féminité ». Preuve de la retenue dont les instances internationales devront faire preuve à l'égard de Caster Semenya, Santhi Soundarajan avait tenté par la suite de se suicider.

Une performance corporelle dite « hors norme» et un « doute visuel » porté sur un corps de femme qui échappe aux représentations normées et genrées déclenchent une batterie de tests médicaux-psychologiques, appelés « tests de féminité » qui n'ont pour objectif que de construire des correspondances entre les représentations du corps, de l'identité du sexe et du genre.

5 J'ai été attirée par cet événement lorsque je travaillais sur le texte de Foucault « le vrai sexe», son commentaire des mémoires d'Herculine Barbin. Et ce sont 
précisément les correspondances qui m’ont intéressée. Dans Naissance de la clinique, Michel Foucault démontre la construction historique de la médecine moderne. Pour Foucault, la médecine moderne est née de l'instauration du « regard médical ». « Le regard qui voit est un regard qui domine »5. Ce regard porte déjà, selon lui, sur un objet constitué à partir d'une norme. Le malade est l'« objet du regard» et le médecin «sujet du regard», l'institution ayant à charge de légitimer socialement le dispositif et la relation entre le sujet qui regarde et l'objet regardé. Il s'agit, comme l'indique Foucault, non pas de savoir ce qu'est le pouvoir, mais comment il s'exerce et quelles catégories de sujets il met en place, avec quelles valeurs, quels intérêts et quelles stratégies. Ainsi qu'il l'écrit dans La Volonté de savoir : « Cette forme de pouvoir demande pour s'exercer des présences constantes, attentives, curieuses aussi ; elle requiert un échange de discours, à travers des questions qui extorquent des aveux et des confidences qui débordent les interrogations [...]. De cela la médicalisation de l'insolite sexuel est à la fois l'effet et l'instrument [...]. Le pouvoir qui, ainsi, prend en charge la sexualité se met en devoir de frôler les corps, il les caresse des yeux, il en intensifie des régions ; il électrise des surfaces ; il dramatise des moments troubles ${ }^{6}$. »

Afin de mettre en perspective «la médicalisation de l'insolite sexuel», je voudrais me pencher sur les Mémoires d'Herculine Barbin, qui datent du milieu du 19ème siècle, à la lumière de ce que l'aventure de Caster Semenya a mis en évidence. Comme l'écrit Foucault dans L'Ordre du discours, « Il faut concevoir le discours comme une violence que nous faisons aux choses, en tout cas comme une pratique que nous leur imposons ${ }^{7}$. »

Les mémoires d'Herculine Barbin ont été retrouvées par Ambroise Tardieu, médecin légiste très connu à son époque et déjà célèbre pour son ouvrage fort remarqué, Les Attentats aux mours ${ }^{8}$. Dans ce livre, véritable guide descriptif anatomique, Tardieu identifie une pratique fréquente de la fellation - pratique hautement réprouvée - à partir de la forme de la bouche d'un individu. Le médecin marche main dans la main avec le juge. Le sexe est d'emblée potentiellement le vecteur du crime. «Dans tous les cas les signes physiques constituent d'excellents moyens pour diriger les recherches de la justice ${ }^{9}$. »

Ambroise Tardieu invente un corps en fonction de la sensibilité de son temps et, ainsi que l'écrit Georges Vigarello qui présente le livre : « Il faut insister sur cette anatomie "imaginée". Elle est sous-tendue par la volonté dejuger plus que de montrer, mais elle pose un problème plus large que celui du recensement des blessures ou celui du soupçon porté sur la victime. Elle répond à une visée d'identification des individus [...]. Une façon d'aider à démasquer les suspects [...]. Il s'agit d'une attente sociale, une inquiétude précise débordant nettement les seuls effets d'agression, une obsession même développée comme jamais avec la société du XIX ${ }^{e}$ siècle et l'abandon des anciens ordres : mieux identifier les individus rendus "indistincts" par le brouillage supposé des conditions, mieux les localiser, mieux les surveiller. Le corps "nu" devient un repère interminablement épié, soumis à la sagacité du médecin légiste puisque l'habit ne suffirait plus à révéler l'origine sociale et l'état de chacun ${ }^{10}$. » S'ouvre l'ère du soupçon sur les pratiques sexuelles que l'on identifie par les formes et les conformations des corps.

C'est en 1874 que le médecin Ambroise Tardieu rend public un ouvrage scientifique intitulé Question médico-légale de l'identité dans ses rapports avec les vices de conformation des organes sexuels, qui comprend un manuscrit découvert en 1868, dans une mansarde du Quartier Latin. Auprès de ce manuscrit il y a un corps : celui d'Abel Barbin, âgé de vingt-huit ans, qui 
venait de se suicider. Abel est né(e) Adélaïde Herculine Barbin (surnommée Alexina par ses proches) et rebaptisé(e) à vingt et un ans, après qu'un tribunal la/le déclare de sexe masculin. Cette décision administrative tient compte de la «prédominance évidente du sexe masculin» d'Abel d'un point de vue physiologique. C'est en 1868 que ce "jeune homme», employé dans une administration de chemin de fer, se donne la mort par asphyxie carbonique. Sur la table, il laisse une lettre adressée à sa mère ainsi qu'un manuscrit intitulé Mes souvenirs ${ }^{11}$, où il raconte sa vie solitaire et misérable.Le médecin de l'état civil chargé de faire le constat de décès fait l'examen du cadavre et ausculte les organes génitaux afin de voir si l'individu n'était pas atteint d'une maladie qui aurait pu expliquer son geste suicidaire. Lors de cet examen, le Dr Régnier remarque avec étonnement « un cas d'hermaphrodisme masculin des mieux caractérisés ${ }^{12}$ ». Ambroise Tardieu peut écrire : «Le fait extraordinaire qui me reste à rapporter fournit en effet l'exemple le plus cruel et le plus douloureux des conséquences fatales que peut entraîner une erreur commise dès la naissance dans la constitution de l'état civil. On va voir, la victime d'une semblable erreur, après vingt ans passés sous les habits d'un sexe qui n'est pas le sien, aux prises avec une passion qui s'ignore elle-même, avertie enfin par l'explosion de ses sens, puis rendue à son véritable sexe en même temps qu'au sentiment réel de son infirmité physique, prenant la vie en dégoût et y mettant fin par le suicide. Ce pauvre malheureux, élevé dans un couvent et dans des pensionnats de jeunes filles jusqu'à l'âge de vingt-deux ans, admis aux examens et pourvu du diplôme d'institutrice, vit à la suite des circonstances les plus dramatiques et les plus émouvantes son état civil réformé par un jugement du tribunal de La Rochelle, et ne put supporter l'existence misérable que son nouveau sexe incomplet lui imposa. Certes, dans ce cas, les apparences du sexe féminin ont été poussées bien loin, et cependant la science et la justice furent contraintes de reconnaître l'erreur et de rendre le jeune homme à son sexe véritable [...]. Je n'hésite pas à publier presque en entier [les mémoires d'Herculine Barbin], ne voulant pas laisser perdre le double et précieux enseignement qu'il renferme, d'une part au point de vue de l'influence qu'exerce sur les facultés affectives et sur les dispositions morales la malformation des organes sexuels, d'une autre part au point de vue de la gravité des conséquences individuelles et sociales que peut avoir une constatation erronée du sexe de l'enfant qui vient de naître ${ }^{13}$. »

Ainsi, c'est pour faire la démonstration scientifique des effets tragiques dus à une erreur d'assignation sexuelle et, surtout, pour établir un lien de causalité «rationnelle» et «scientifique» entre appartenance sexuelle et attirance sexuelle que Tardieu publie Mes Souvenirs d'Herculine Barbin.

Herculine est élevée dans des institutions religieuses, fille parmi d'autres. À propos de sa réassignation de genre, elle écrit : « Ce qui s'était passé ne fut pas pour moi une révélation, mais un tourment de plus dans ma vie ${ }^{14}$. » Loin, donc, d'être une révélation qui la délivrerait d'un « mal » identitaire, cette véridiction lui cause bien plus de souffrance que de soulagement. Et Foucault de commenter: "Les souvenirs de cette vie, Alexina les a écrits une fois découverte et établie sa nouvelle identité. Sa "vraie" et "définitive" identité. Mais il est clair que ce n'est pas du point de vue de ce sexe enfin retrouvé qu'elle écrit. Ce n'est pas l'homme qui parle enfin, essayant de se rappeler ses sensations et sa vie du temps qu'il n'était pas encore "lui-même". Quand Alexina rédige ses mémoires, elle n'est pas très loin de son suicide; elle est toujours pour elle-même sans sexe certain, mais elle est privée des délices qu'elle éprouvait à n'en pas avoir ou à n'avoir pas tout à fait le même que celles 
au milieu desquelles elle vivait, et qu'elle aimait, et qu'elle désirait si fort ${ }^{15}$. » Pourtant, Alexina n'est pas « sans sexe certain » quand elle aime et qu'elle désire. Foucault passe sans doute un peu vite sur les modalités d'énonciation du texte d'Alexina. Même après sa réassignation de sexe par l'état civil, Alexina écrit ses souvenirs dans le genre grammatical féminin. En effet, Alexina, dans le langage qui la soutient, dans le corps de son écriture, aime en femme et aime les femmes: "J'étais généralement aimée [c'est elle qui souligne] de mes maîtresses et de mes compagnes, et cette affection, je la leur rendais bien. J 'étais née pour aimer. [...]J e me liais bientôt d'une étroite amitié avec une charmante jeune fille nommée Thécla, plus âgée que moi d'une année. Certes, rien n'était plus opposé extérieurement que notre physique. Mon amie était aussi fraîche, aussi gracieuse que je l'étais peu. On ne nous appela que les inséparables, et, en effet, nous ne nous perdions pas de vue d'un seul instant ${ }^{16}$. »Née pour aimer, aimée de ses compagnes. C'est aussi ce désir et ces élans érotiques qui deviennent suspects pour le médecin et le juge de l'époque. C'est parce qu'en tant que femme elle désire une femme qu'Alexina est assignée par le corps médical à être un homme. À qui appartient un corps dans ce contexte? À la grammaire du sujet ou à la subjectivité d'une époque? Pour Tardieu et les savants de l'époque: une femme qui aime et qui désire une femme ne peut être qu'un homme.

Puis Alexina entretient une relation amoureuse et sexuelle passionnée avec la jeune Sara: "Dans nos délicieux tête-à-tête, elle se plaisait à me donner la qualification masculine, que devait plus tard, m'accorder l'état civil ${ }^{17}$. » Dans le récit de ses jeux érotiques - sa compagne la nomme Camille dans leurs élans amoureux et sexuels - et de sa passion amoureuse partagée pour son amie Sara, Alexina déstabilise la langue, défait et perturbe la hiérarchie des genres, passant du genre masculin au genre féminin et le fait en toute conscience. C'est elle-même qui souligne les modifications de genre grammatical. "Je priai un soir mon amie de partager mon lit. Elle accepta avec plaisir. Dire le bonheur que je ressentis de sa présence à mes côtés, serait chose impossible! J 'étais folle de joie ! Nous causâmes longuement avant de nous endormir, moi les deux bras passés autour de sa taille, elle, reposant, le visage près du mien! Mon dieu ! Ai-je été coupable? Et dois-je donc ici m’accuser d'un crime? Non, non ! Cette faute ne fut pas la mienne, mais celle d'une fatalité sans exemple, à laquelle je ne pouvais résister !!! Sara m’appartenait désormais !! Elle était à moi !! Ce qui, dans l'ordre naturel des choses, devait nous séparer dans ce monde, nous avait unis !! Qu'on se fasse, s'il est possible, une idée de notre situation à tous deux ${ }^{18 ! ~ » ~}$

«Folle de joie» dans l'élan érotique et passionné qui la lie à Sara, Alexina, rappelée au monde dans lequel elle vit, déplore et s'inquiète de la situation de tous deux. Élevée femme, instruite femme, « construite socialement femme » si je puis dire, vivant dans une communauté de femmes, elle rapporte les effets catastrophiques sur sa vie et son corps de la « vérité médicale» de son sexe indéterminé. Les pratiques sexuelles sont ici rabattues sur la nécessité d'avoir un vrai sexe et de faire correspondre l'appartenance sexuelle à l'attirance sexuelle. Alexina est médicalement hermaphrodite, pourtant les conclusions du rapport d'expertise médicale affirment la « prédominance évidente du sexe masculin ». « Des faits ci-dessus que conclurons-nous? Alexina est-elle une femme? Elle a une vulve, de grandes lèvres, un urètre féminin. Ce sont là des attributs tout féminin : oui, mais Alexina n'a jamais été réglée; tout l'extérieur du corps est celui d'un homme, mes explorations n'ont pu me faire trouver la matrice. Ses goûts, ses penchants l'attirent vers les femmes. Voilà les vrais 
témoins du sexe; nous pouvons à présent conclure et dire: Alexina est un homme, hermaphrodite sans doute, mais avec prédominance évidente du sexe masculin ${ }^{19}$. »

Les vrais témoins du sexe sont donc les penchants qui l'attirent vers les femmes. Le désir érotique, tel est l'incontrôlable. Et ainsi que l'écrit Georges Canguilhem dans son article « La monstruosité et le monstrueux » : « Sous une forme rationalisée, donc affaiblie, nous retrouvons bien le monstrueux à l'origine des monstruosités ${ }^{20}$. » Le discours rationnel de la science médicale tend à démontrer en quoi des conformations physiques anormales sont l'indice de potentialités monstrueuses au plan social. Le monstrueux porte en lui le délictueux.

8 Alors, comme l'écrit encore Canguilhem, « en présence d'un oiseau à trois pattes, faut-il être plus sensible à ceci que c'est une de trop ou à cela que ce n'est guère qu'une de plus ${ }^{21}$ ? »

Mais que se passe-t-il lorsque Alexina a été rendue à « son vrai sexe » ? Que se passe-t-il lorsque le discours social et la société rendent conformes une conformation sexuée et un désir sexuel ? Ce qui pouvait permettre à Abel d'aimer son amie Sara, lors du changement de nom et de sexe dans l'état civil d'Herculine Barbin, sans plus se cacher, sans plus ressentir de honte et d'angoisse, devient le point de bascule vers le suicide. Le «vrai sexe» d'Herculine, établi par le discours social et scientifique, son identité sexuelle rendue à sa « véridiction » la/le conduit au suicide.

Elle écrit: «C'en était donc fait. L'état civil m’appelait à faire partie désormais de cette moitié appelée sexe fort. Moi, élevé jusqu'à l'âge de vingt et un ans dans les maisons religieuses, au milieu de compagnes timides, j'allais comme Achille laisser loin derrière moi tout un passé délicieux et entrer dans la lice, armé de ma seule faiblesse et de ma profonde inexpérience des hommes et des choses $^{22}$ ! » J eune fille parmi les jeunes compagnes timides, elle devient Achille à la fragilité déniée.

Le fait d'appartenir à l'univers masculin, appartenance qui est désormais marquée par l'énonciation dans le genre masculin - alors qu'Alexina avait habitué son lecteur à un brouillage des genres, ou qu'elle soulignait le genre féminin - , ne fabrique pas de correspondance avec son « véritable sexe ».

9 Ce sont précisément ces passages de souffrance que Tardieu s'emploie à censurer, laissant quelques pages pour ne pas rompre l'élan de ce style d'écriture que Foucault considère comme "une manière de vivre», un ton d'écriture qui sont aussi des façons d'être au monde: "ce style élégant, apprêté, allusif, un peu emphatique et désuet qui était pour les pensionnats d'alors non seulement une façon d'écrire, mais une manière de vivre ${ }^{23}$ ». C'est pourquoi Alexina peut écrire: "Cette lutte incessante de la nature contre la raison m'épuise chaque jour davantage et m'entraîne à grands pas vers la tombe. [...]. Ce jour arrivé, quelques médecins feront un peu de bruit autour de ma dépouille; ils viendront en briser tous les ressorts éteints, y puiser de nouvelles lumières, analyser toutes les mystérieuses souffrances amassées sur un seul être. Ô princes de la science, chimistes éclairés, dont les noms retentissent dans le monde, analysez donc, s'il est possible, toutes les douleurs qui ont brûlé, dévoré ce cœur jusque dans ses dernières fibres; toutes ces larmes brûlantes qui l'ont noyé, desséché sous leurs sauvages étreintes !24 »

Alexina avait donc raison d'écrire qu'elle allait devenir l'objet du regard et du langage de la science, mais non pas, comme elle le dit, pour « analyser toutes les douleurs qui ont brûlé, dévoré ce coeur jusque dans ses dernières fibres », mais pour ouvrir, disséquer, porter un regard expert sur le réel d'un corps : les 
médecins essayant d'atteindre " l'idéal d'une description exhaustive ». Cette recherche d'image, cette volonté de voir a été tellement recherchée que Félix Nadar, le photographe de l'époque, a fait des clichés d'hermaphrodites. Certains de ces clichés seraient le portrait d'Herculine Barbin. ${ }^{25}$ Cette volonté d'exactitude de description du réel repose sur le postulat que, comme l'écrit Foucault, « tout le visible est énonçable et qu'il est tout entier visible parce que tout entier énonçable 26 ».

La médecine corrige le tort fait au sujet, et Tardieu et ses savants confrères ne remirent jamais en question le bien-fondé de l'acte de rectification du sexe d'Herculine. Ainsi que l'écrit Canguilhem: «Le propre d'une fausse science c'est de ne rencontrer jamais le faux, de n'avoir renoncé à rien, de n'avoir jamais à changer de langage. Pour une fausse science, il n'y a pas d'état préscientifique. Le discours de la fausse science ne peut pas recevoir de démenti. Bref la fausse science n’a pas d'histoire27. » Précisément, ce que coupe Tardieu, c'est l'histoire et le récit des souffrances d'Abel, ex-Alexina, il passe sous silence la parole et le récit écrit après le changement d'état civil. L'histoire du sujet ne l'intéresse pas. Ici, seule la maladie parle et exige du médecin sa parole savante.

11 On peut donc constater que dans ce contexte la sexualité et les pratiques sexuelles sont l'objet et l'enjeu d'un discours scientifique qui détermine l'identité d'un sujet : tout se passe comme si la vérité d'un sujet était réductible à son identité sexuelle. Selon Foucault, avec la sexualité et son dispositif discursif, le sujet est devenu un « homme psychologique». Alors « avons-nous vraiment besoin d'un vrai sexe?» telle est la question quelque peu provocatrice que pose le philosophe dans son texte de présentation à l'édition d'Herculine Barbin dite Alexina B. ${ }^{28}$.

La vérité n'est-elle pas alors au service d'une catégorisation du moi que le sujet intériorise lui-même pour être identifié, reconnu dans le regard de l'autre? La sexualité a été l'un des champs soumis à ce régime de véridiction afin de constituer l'identité et la personnalité d'un sujet. Et la médecine moderne s'est introduite dans ce champ de véridiction.

Le doute visuel pour Caster Semenya ou le doute sexuel pour Alexina font vaciller un discours scientifique qui prétend à «la vérité du sexe». La normalisation d'un corps passe par la reconnaissance visible d'une appartenance à un sexe et un seul.

Michel Foucault écrit: «On a mis bien longtemps à postuler qu'un hermaphrodite devait avoir un seul, un vrai sexe».

Au Moyen Âge, développe-t-il, c'était le rôle du père ou du parrain (de ceux, donc, qui "nommaient" l'enfant), de fixer, au moment du baptême, le sexe qui allait être retenu. Puis à partir du XviII siècle, les théories biologiques de la sexualité ont conduit peu à peu à refuser l'idée d'un mélange des deux sexes en un seul corps et à restreindre par conséquent le libre choix des individus incertains. «Désormais, à chacun un sexe, et un seul. À chacun son identité sexuelle première, profonde, déterminée et déterminante. » Du point de vue médical, il s'agit désormais de déchiffrer quel est le vrai sexe qui se cache sous des apparences. Et Foucault de commenter : «J e sais bien que la médecine du $\mathrm{XIX}^{\mathrm{e}}$ siècle et du $\mathrm{XX}^{\mathrm{e}}$ a corrigé beaucoup de choses dans ce simplisme réducteur [...]. Pourtant, l'idée qu'on doit bien avoir finalement un vrai sexe est loin d'être tout à fait dissipée. Quelle que soit sur ce point l'opinion des biologistes, on trouve au moins à l'état diffus, non seulement dans la psychiatrie, la psychanalyse, la psychologie, mais aussi dans l'opinion courante, l'idée qu'entre sexe et vérité il existe des relations complexes, obscures et essentielles. On est, c'est certain, plus tolérant à l'égard des pratiques qui transgressent les 
lois. Mais on continue à penser que certaines d'entre elles insultent à "la vérité" [...] on est disposé peut-être à admettre que ce n'est pas une grave atteinte à l'ordre établi ; mais on est assez prêt à croire qu'il y a là quelque chose comme une "erreur". Une "erreur" entendue au sens le plus traditionnellement philosophique: une manière de faire qui n'est pas adéquate à la réalité; l'irrégularité sexuelle est perçue peu ou prou comme appartenant au monde des chimères ${ }^{29}$. »

Dans ses travaux, Michel Foucault étudie le discours sur le sexe qui établit historiquement le lien entre la sexualité, la subjectivité et l'obligation de vérité, une triade qu'il verse au compte de la psychanalyse comme héritière de cette scientia sexualis qui cherche la vérité au fond du sexe ; et qui serait supposée vouloir dire le vrai sexe et l'identité par l'usage de la norme, accompagnée d'une pratique de l'aveu. «Et puis on admet aussi que c'est du côté du sexe qu'il faut chercher les vérités les plus secrètes et les plus profondes de l'individu ; que c'est là qu'on peut le mieux découvrir ce qu'il est et ce qui le détermine; et si pendant des siècles on a cru qu'il fallait cacher les choses du sexe parce qu'elles étaient honteuses, on sait maintenant que c'est le sexe luimême qui cache les parties les plus secrètes de l'individu : la structure de ses fantasmes, les racines de son moi, les formes de son rapport au réel. [...] $\mathrm{Au}$ point de ce croisement de ces deux idées - qu'il ne faut pas nous tromper en ce qui concerne notre sexe, et que notre sexe recèle ce qu'il y a de plus vrai en nous - , la psychanalyse a enraciné sa vigueur culturelle. Elle nous promet à la fois notre sexe, le vrai, et toute cette vérité de nous-même qui veille secrètement en luii ${ }^{30}$. »

Pourtant, Freud n'a eu de cesse de démontrer que l'enfant est un «pervers polymorphe» et que la sexualité infantile est le terreau de la vie psychique. L'ambiguïté du sexuel, l'étrangeté et la perversion polymorphe de l'invention freudienne sont recouverts par les discours de catégorisation, de normalisation. Foucault critique dans la médecine - relayée selon lui par la psychanalyse toute la violence des dispositifs de pouvoirs qui tendent à établir la vérité d'un corps ou à caractériser le corps en vérité. Pour lui, le corps excède et déséquilibre la vérité par le plaisir, irréductible à toute détermination ou toute catégorisation. Les corps et les plaisirs, ainsi qu'il l'écrit dans La Volonté de savoir, ont charge de rappeler le caractère précaire, contestable et potentiellement violent de toute caractérisation du corps en vérité. Les corps et les plaisirs doivent être préservés, défendus et affirmés contre toute prétention à déterminer définitivement ce qu'il en est du corps.

L'analyse qu'il propose du mémoire rédigé par Herculine est en effet traversée par une opposition qu'il établit entre la différence sexuelle qui est le marqueur d'une identité subjective, àlaquelle Herculine est assignée, et les différences corporelles qui relèvent de l'expérience et de ses pratiques.

Malgré les mises en ordre sociales et discursives qui tentent de l'assigner, de le catégoriser, le corps par l'expérience des plaisirs qui le rendent indéfinissable fait acte de résistance. En tant que pratique, la sexualité échappe à toute définition unique et laisse au corps l'ouverture de sa pluralité.

Ce refus foucaldien d'identifier un individu par et à travers sa sexualité, J ean Allouch le prolonge d'une devise : «Il n'y a pas de vérité du sexe. » ou encore « chacun est une minorité sexuelle»31.

Pourtant, et « il faut être juste avec Freud », pour reprendre une expression de Foucault lui-même, même si la psychanalyse dans ses développements discursifs a délimité les contours de la sexualité dans les normes de son temps, il faut reconnaître à Freud d'avoir théorisé la perversion polymorphe, la 
sexualité infantile, l'inconscient qui ne connaît pas la différence des sexes et avec tout cela le concept de pulsion. J e le rappelle, la pulsion n'a pas d'objet ou de but prédéterminé. Freud écrit dans les Trois essais sur la théorie du sexuel en 1915 : « Selon la manière de voir de la psychanalyse, l'intérêt sexuel exclusif de l'homme pour la femme est donc également un problème nécessitant éclaircissement et pas une chose allant de soi, pour laquelle on pourrait présupposer à la base une attirance chimique ${ }^{32}$. »

L'élan érotique d'Alexina la menait vers les femmes, était-ce suffisant pour déterminer son «vrai sexe»? Lorsque Alexina devient Abel, il rompt sa relation avec son amie Sara, c'est là que la pulsion peut faire entendre quelque chose qui fait obstacle, scandale donc pour l'époque. C'est sans doute à cette écoute que le praticien freudien peut se délester de ses propres normes et discours. Rompre alors avec l'idéal d'un certain savoir, contourner les « idéauxlogies », se déprendre du discours du Maître.

Pour conclure je voudrais citer Marie-J osé Mondzain : «Il y a des visibilités qui personnifient un discours, c'est toujours le discours du maître. Dès lors, le visible endoctrine et incorpore le spectateur à la visibilité d'un corps personnifiant, qui n'est autre que le corps du discours qui le sous-tend. Le discours du maître soumet le regard au visible et l'engloutit dans l'assentiment. Tout autres sont les visibilités dont la forme ne personnifie rien et qui sont habitées par la parole [...]. Dans ce cas le visible met le spectateur dans une place où l'image reste à construire ${ }^{33}$ »

Le « doute visuel », les tests médicaux, l'absence de parole singulière, telles ont été les pratiques des Tardieu et autres médecins légistes. Le regard qui scrute un corps a comme visée de lui donner une signification sexuelle, une identité sexuée. Constater l'erreur, tel est le projet « médico-juridique» des Tardieu actuels ou de l'époque, établir un régime de véridiction qui dit non seulement ce qui est, mais ce qui doit être du désir, des pratiques corporelles ou sexuelles. La bizarrerie d'un comportement par l'attirance sexuelle ou par la performance corporelle produit une visibilité, une représentation imaginaire et le corps est alors la proie d'un visible captif de l'imaginaire.Il reviendrait au discours de l'analyse de soumettre au jeu du questionnement « le dynamisme du travail de la vérité 34 » - selon l'expression de Lacan - , sa part conflictuelle et dialectique. Ainsi que l'écrit Lacan, le discours analytique se spécifie, se distingue « de poser la question d'à quoi sert cette forme de savoir, qui rejette et exclut la dynamique de vérité35 ». La dynamique c'est-à-dire sa potentialité conflictuelle, celle d'un non-savoir.

\section{Notes}

1 FoUCAULT Michel (2001), «Sexualité et pouvoir » (1978), dans Dits et Écrits, t. II, Paris, Gallimard, coll. « Quarto », p. 522-531, p. 528.

2 DAVIDSON Arnold I., L'Émergence de la sexualité, op. cit., p. 9.

3 Ibid., p. 11.

4 C'est moi qui souligne.

5 FOUCAULT Michel (1963), Naissance de la clinique, Paris, PUF, p. 38.

6 ID. (1976), La Volonté de savoir, t. I d'Histoire de la sexualité, Paris, Gallimard, p. 6061.

7 FOUCAULT Michel (1971), L'Ordre du discours (1970), Paris, Gallimard, p. 55.

8 TARDIEU Ambroise (1995), Les Attentats aux moeurs (1857), Grenoble, J érôme Millon.

9 Ibid., p. 389. 
10 VigARELLO Georges, «La violence sexuelle et l'œil du savant», dans TARDIEU Ambroise, Les Attentats aux mœurs, op. cit., p. 23-24.

11 Tardieu publia ce manuscrit dans Question médico-légale dans ses rapports avec les vices de conformation des organes sexuels (1872), Paris, Baillière. Tardieu ne publia pas l'intégralité du manuscrit: "Manque, d'abord et surtout, une partie des souvenirs d'Alexina. Tardieu semble avoir reçu le manuscrit complet des mains du médecin, le docteur Régnier, qui avait fait le constat de décès et pratiqué l'autopsie. Il l'a gardé, ne publiant que la partie qui lui paraissait importante. Il a négligé les souvenirs des dernières années d'Alexina - tout ce qui, selon lui, n'était que plaintes, récriminations et incohérences. Malgré les recherches, il n’a pas été possible de retrouver le manuscrit que Tardieu a eu entre les mains. » (FOUCAULT Michel, dans BARBIN Herculine, Mes souvenirs, op. cit, p. 131.

12 GouJ ON Dr E., « Étude d'un cas d'hermaphrodisme imparfait chez l'homme », dans BARBIN Herculine, Mes souvenirs, op. cit., p. 142.

13 TARDIEU Ambroise, dans BARBIN Herculine, Mes souvenirs, op. cit., p. 137. C'est moi qui souligne.

14 BARBIN Herculine, Mes souvenirs, op. cit., 41.

15 FOUCAULT Michel, « Le vrai sexe », art. cité, p. 939 ; je souligne.

16 BARBIN Herculine, Mes souvenirs, op. cit., p. 35.

17 Ibid., p. 68.

18 Ibid., p. 61.

19 CHESNET Hippolyte, Question d'identité ; vice de conformation des organes génitaux externes ; hypospadias ; erreur sur le sexe, rapporté par Michel Foucault dans BARBIN Herculine, Mes souvenirs, op. cit., p. 142, c'est moi qui souligne.

20 CANGUILHEM Georges (2003), La Connaissance de la vie (1965), Paris, Vrin, p. 225.

21 CANGUILHEM Georges, La Connaissance de la vie, op. cit., p. 222.

22 BARBIN Herculine, Mes souvenirs, op. cit., p. 102.

23 FOUCAULT Michel, « Le vrai sexe », art. cité, p. 938.

24 BARBIN Herculine, Mes souvenirs, op. cit., p. 116-117.

25 J e renvoie au livre de Magali LE MENS et J ean-Luc NANCY (2009), L'hermaphrodite de Nadar, Paris, Créaphis Éditions.

26 FOUCAULT Michel, Naissance de la clinique, op. cit., p. 116.

27 CANGUILHEM Georges (2009), « Qu'est-ce qu'une idéologie scientifique ? », Idéologie et rationalité dans l'histoire des sciences de la vie (1977), Paris, Vrin, p. 39-55, p. 46.

28 ID. (2001), «Le vrai sexe», dans Dits et Écrits, t. II, Paris, Gallimard, coll. «Quarto », p. 934-942, p. 935.

29 Ibid., p. 935.

30 FOUCAULT Michel, « Le vrai sexe », art. cité, p. 937.

31 ALLOUCH J ean (2003), «Lacan et les minorités sexuelles », Cités $n^{\circ} 16$, Jacques Lacan, psychanalyse et politique, p. 71-77.

32 FREUD Sigmund (1905), Trois essais sur la théorie sexuelle, tr. fr. (1987), Paris, Gallimard, p. 51.

33 MondZAIn Marie-J osé (2003), L'image peut-elle tuer ? Paris, Bayard, p.61.

34 Ibid., p. 103.

35 Ibid. J e souligne.

\section{Pour citer cet article}

Référence électronique

Laurie Laufer, «La fabrique du corps sexué », Recherches en Psychanalyse [En ligne],

10 | 2011, mis en ligne le 17 janvier 2011, Consulté le 22 janvier 2011. URL :

http://recherchespsychanalyse.revues.org/index1298.html 


\author{
Auteur \\ Laurie Laufer \\ Maître de Conférences HDR, UFR SHC, Paris 7 Denis-Diderot, CRPMS, 40 rue du \\ banquier 75013 Paris. \\ Articles du même auteur \\ La morgue : voir l'irreprésentable [Texte intégral] \\ Paru dans Recherches en Psychanalyse, 8| 2009
}

\title{
Droits d'auteur
}

Tous droits réservés 